\title{
Jaffe-Lichtenstein syndrome - Report of a rare case
}

\author{
Shaul Hameed .K ${ }^{1, *}$, Laxmikant Chatra ${ }^{2}$, Prasanth Shenoy ${ }^{3}$, Anoop Kurian Mathew ${ }^{4}$ \\ ${ }^{\mathbf{1}}$ Assistant Professor, ${ }^{2}$ Senior Professor and HOD, ${ }^{3}$ Senior Professor, ${ }^{4}$ Reader, Dept. of Oral Medicine and Radiology, ${ }^{\mathbf{1} C o l l e g e ~ o f ~ D e n t i s t r y, ~}$ \\ Qassim University, Saudi Arabia, ${ }^{2,3}$ Yenepoya Dental College, Yenepoya University, Mangalore, Karnataka, ${ }^{4}$ Indira Gandhi Institute of \\ Dental Sciences, Kerala, India
}

*Corresponding Author: Shaul Hameed .K

Email: dr.shaul.hameed@qudent.org

\begin{abstract}
Fibrous dysplasia (FD) is a benign slow growing skeletal disorder that causes swelling of the bone. Lichtenstein described FD in 1938 as skeletal bone disorder which is distinguished by continuous replacement of normal bone with fibrous matrix. FD is also associated with Jaffe-Lichtenstein syndrome (JLS) and McCune-Albright syndrome (MAS). JLS is characterized by polyostotic FD along with café-au-lait pigmented lesions on the skin. MAS presents with additional features of endocrine disorders along with the FD. The disease is found to cause significant aesthetic problem and functional disturbance, particularly those affecting the craniofacial skeleton. Most often, the clinical features and the classic radiographic presentation of the pathology would be sufficient to make a diagnosis. However other fibro-osseous lesions that mimic the radiographic features are critical to make a differential diagnosis for the clinician. The present case report is about a 6 year old boy from south India who has been diagnosed as Jaffe-Lichtenstein syndrome (JLS).
\end{abstract}

Keywords: Fibrous dysplasia, McCune-Albright Syndrome, Cafe-au-laithyperpigmentation, Lichtenstein-Jaffe's disease, Osteitisfibrosa, alkaline phosphatase, Acid phospatase, Bone biopsy.

\section{Introduction}

Fibrous dysplasia (FD) is a hamartomatous fibroosseous disease of the bone which was first described by Von Recklinghausen as "Osteitis Fibrosa Generalisata" in 1891 and was later termed as "fibrous dysplasia" by Lichtenstein in the year $1938 .{ }^{1}$ The disease is a relatively rare entity with an incidence rate of 1:4000-1:10,000 and is comprised of $2.5 \%$ of all the bony lesions and about $7 \%$ of the cases are benign bone tumors. ${ }^{2,3}$ It is characterized by replacement of bony architecture by a mixture of fibrous and osseous elements and has been described in three subtypes which are termed as monostotic, polyostotic and craniofacial fibrous dysplasia. Craniofacial fibrous dysplasia (CFD) is confined to the craniofacial skeleton in which maxilla is more often affected than the mandible with a unilateral involvement. Craniofacial skeleton is a complex structure which has multiple adjacent bones; and hence the craniofacial fibrous dysplasia cannot be grouped under Monostotic type of fibrous dysplasia. Most often they are found in the first three decades of life and become static when the patient's skeletal maturity is complete. ${ }^{3}$ The disease is usually gradual in onset and presents with the painless enlargement of the involved bone. If the disease is affected in the craniofacial region it may lead to asymmetry of the face. ${ }^{2}$ Malformed extremity, pain in the limb or pathologic fracture may be seen if the long bones are affected. If the disease causes obliteration of bony cavities it may lead to loss of hearing, tinnitus, nasal obstruction, facial paralysis etc. Diagnosis of the disease is completely based on the effective evaluation of the clinical, radiographic and histopathological examinations.

\section{Case Report}

A six-year-old boy reported to the department of Oral medicine and radiology, Yenepoya Dental College with a chief complaint of swelling in the lower jaw and right side of the nose since 2 years. (Fig. 1) History revealed that initially the swelling was smaller in size, gradual in onset, and hasattained the present size gradually during the course of last two years. The swelling was completely asymptomatic. However, the patient had reported mild hearing loss and reduced smell sensation. Patient's past medical history was non-contributory and the family history did not reveal any similar kind of problem.

On general physical examination, the patient was moderately built and nourished, conscious and cooperative, and the vital signs were within the normal limit. A café-aulait macule measuring $15 \times 12 \mathrm{~cm}$ was noted on the right lateral side of the neck. (Fig. 2)

On extra oral examination, gross facial asymmetry with ballooning enlargement of the mandible and a solitary swelling on the right side of the nose is noted (Fig. $1 \& 3$ ). On detailed local examination, there was a smooth swelling measuring approximately $6 \times 10 \mathrm{~cm}$ involving the mandible that extends from the right ramus of the mandible till the left angle of the mandible. Ballooning of the cortex could be appreciated for the swelling. The right side of the nose showed another solitary swelling that is measuring approximately $1.5 \mathrm{~cm}$ in diameter. (Fig. $1 \& 3$ ) Skin over the swelling appeared normal. On palpation swelling was bony hard in consistency, smooth, non-tender with no local rise in temperature.

Intra oral examination revealed diffuse enlargement of the lower alveolar process. There was expansion of the bony cortex giving an increased width for the mandible. Teeth were vital and the surrounding soft tissue appeared to be normal. There was dental caries in the 55, 52, 51, 64, 65 and 74. (Fig. 4) Based on the history and the clinical examination, a provisional diagnosis of Jaffe's type of 
Cranio-facial fibrous dysplasia and dental caries in relation to $55,52,51,64,65$ and 74 were given.

Mc Cune-Albrights syndrome and Paget's disease were considered in the differential diagnosis. Mc Cune-Albrights syndrome is usually a severe form of fibrous dysplasia that exhibits certain endocrine abnormalities which was negative for the present case and Paget's disease usually occurs in maxilla usually in middle or old age people, but our case was 6 year dimension

Panoramic radiograph was advised as part of investigation, which revealed normal component of Temporo-Mandibular Joint with mixed dentition. With respect to the area of interest, mandible appeared to be enlarged in size. Obliteration of the cortex was seen with a smooth curved projection downward for the inferior margin of the mandible the convexity is downwards resembling thumb print pattern (Fig. 5) as if the bone had been soft and preserved up on by thumb, central area of the mandible appears to be alternate area of radio opacity and radiolucency appearing like cotton wool. Para nasal sinus projection (Waters' view) revealed the encroachment of right maxillary antrum and its margin appears to merge with adjacent bone. Ribbon like cortex (Fig. 6) was appreciated. Skeletal radiographic survey showed no abnormality. Considering the radiographic investigation, the condition was diagnosed as fibro-osseous dysplasia of maxilla and mandible.

Bone marrow trephine biopsy was carried out and specimen was sent for histopathological examination, which revealed numerous trabeculae of woven bone with osteoblastic rimming in a fibrous connective tissue stroma suggestive of Fibro-osseous lesion.

Ultrasonography of abdomen revealed mild hydronephrosis on right side and auditory brain stem report showed minimal hearing loss on the left side and hearing sensitivity was within the normal limit in the right ear. No abnormalities were reported in the hematology and Echocardiography examination. Serum alkaline phosphatase was $215 \mathrm{U} / \mathrm{L}$ and serum calcium level was $9.2 \mathrm{mg} / \mathrm{dl}$. Based on the investigation final diagnosis of the Jaffe-Lichtenstein syndrome (JLS) was made and patient was referred to craniofacial unit for the further management.

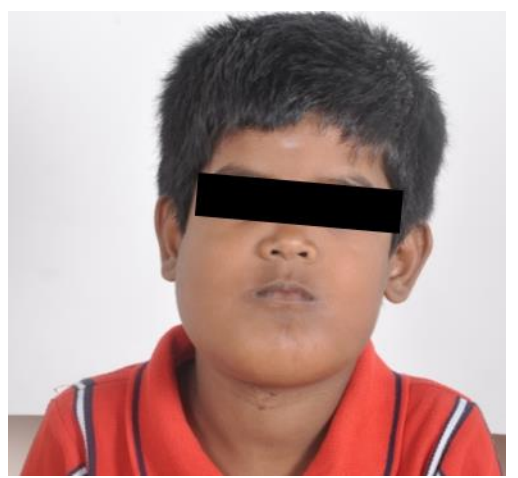

Fig. 1: The extra oral photograph showing gross facial asymmetry due to diffused swelling involving the lower half of the face

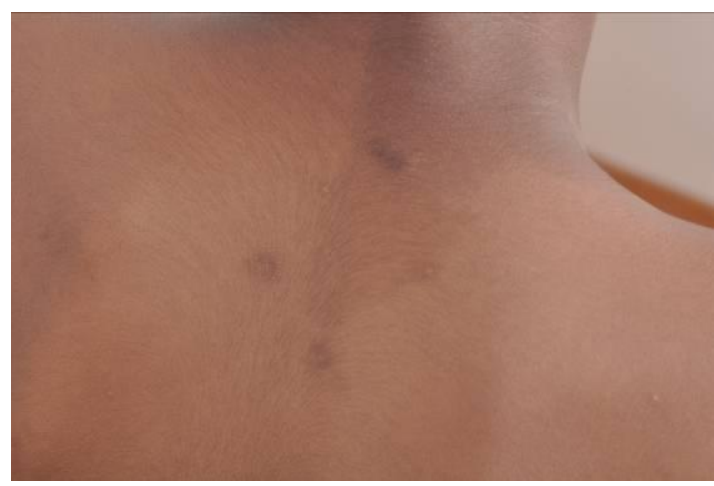

Fig. 2: Photograph showing café-au-lait macule on right side of the neck

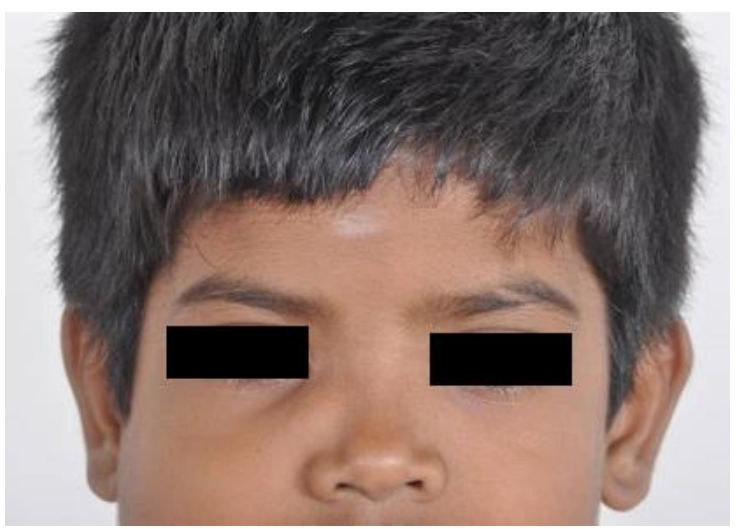

Fig. 3: The extra oral photograph showing a solitary swelling on the right side of the bridge of the nose

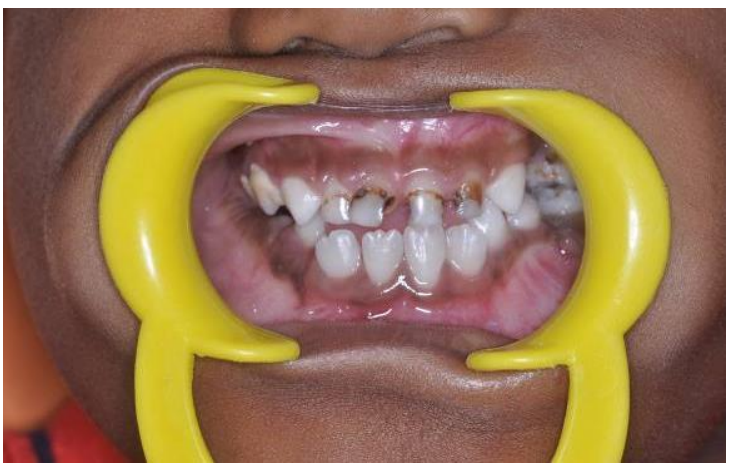

Fig. 4: The photograph showing diffused enlargement of the lower alveolar process

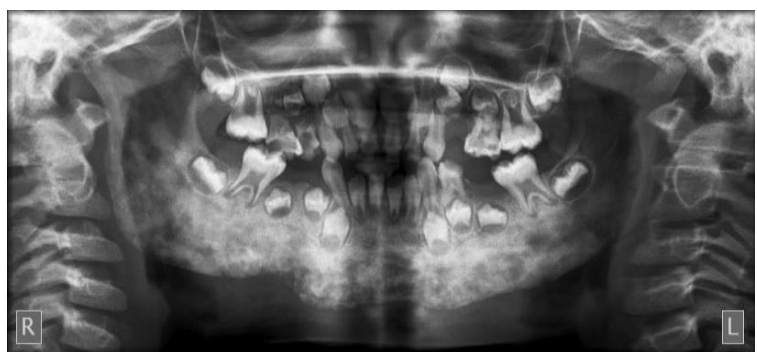

Fig. 5: Panoramic radiograph showing enlarged mandible with altered trabecular pattern having areas of radiopacity and radiolucency giving a cotton wool appearance 


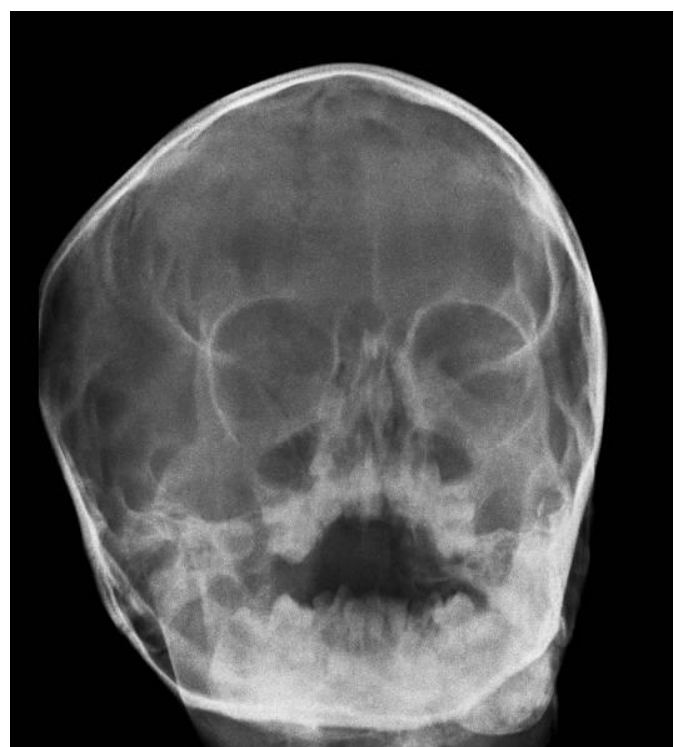

Fig. 6: PNS waters' view shows cotton wool appearance in the mandible and encroachment of right maxillary antrum. The margins of the bony changes appear to be blending with the adjacent bone

\section{Discussion}

The monostotic type of fibrous dysplasia common affects the age group of 20 to 30 years and the polyostotic type FD has seen mainly in children younger than 10 years of age with equal gender distribution. The lesions tend to grow more during childhood and stabilize after reaching puberty. FD commonly affects the craniofacial bones, metaphysis or diaphysis of the proximal femur or tibia and ribs. ${ }^{3}$ The occurrence of polyostotic to monostotic FD is reported to be with a ratio of 3:7.,5 The polyostotic FD is also seen in association with JLS which has characteristic café-au-lait pigmented skin lesions and in MAS with endocrinopathies. ${ }^{1,2}$ The disease is usually asymptomatic but at times the patients may get bone pain and pathological fractures along with bone deformities. ${ }^{6}$ Malignant transformation rate for FD is reported to be very rare. ${ }^{7}$

Craniofacial FD may present with various symptoms like pain, nasal congestion, mal-aligned or displaced teeth and facial asymmetry depending on the location of the lesions. The facial asymmetry can cause bulging of the eyes (proptosis), and difference in the vertical positioning of the eyes (vertical dystopia) and frontal bossing and the facial abnormality can vary greatly from person to person. FD may cause neurological symptoms due to compression of nearby nerves by abnormal tissue development. Some of the specific symptoms include loss of vision and hearing impairment because of the compression of optic and auditory nerves respectively.

The present case was a 6 year old boy with asymptomatic bony hard swelling on the face involving the mandible and right side of the face. In the present case our patient had mild hearing loss and reduced smell sensation. The patient also had café-au-lait pigmentation on the right lateral side of the neck, however there were no endocrine disorders, and hence the provisional diagnosis of JaffeLichtenstein syndrome was given.

A differential diagnosis of $\mathrm{Mc}$ Cune-Albrights syndrome was considered for the present case. It was ruled out at our patient did not show any endocrinal abnormalities. Second differential diagnosis of Paget's disease was made which is slow growing skeletal condition of abnormally rapid bone destruction (osteolytic) and reformation (osteoblastic). ${ }^{8}$ The new bone formation of makes the bone structurally abnormal which may cause bone pain, arthritis, deformities and fractures. ${ }^{9}$ It is commonly found in the elder individuals after 50 years of age. Our case was only 6 years of age and hence Paget's disease was ruled out.

Various radiographic features have been reported in Fibrous dysplasia. The most common being the ground glass pattern which is followed by orange peel appearance. And other features include cotton wool and thumb print appearance..$^{10}$ In the present case, there was a smooth curved projection downward for the inferior margin of the mandible with a downward convexity resembling thumb print pattern.

Serum Alkaline Phosphatase (ALP) is occasionally elevated in some cases, but calcium, parathyroid hormone, 25-hydroxyvitamin D, and 1,25-dihydroxyvitamin D levels in most cases of FD are in normal range. Persons with extensive polyostotic FD may have hypophosphatemia, hyperphosphaturia and osteomalacia. ${ }^{3}$ For the present case the serum alkaline phosphatase was $215 \mathrm{U} / \mathrm{L}$ and serum calcium level was $9.2 \mathrm{mg} / \mathrm{dl}$.

A bone biopsy can reveal characteristic changes to bone that occur in individuals with FD and may be necessary to distinguish a FD lesion from other types of growths or tumors if it is unclear after an $\mathrm{x}$-ray. Bone marrow trephine biopsy showed the classical features of fibro osseous lesion, which helped, in the histopathological confirmation. Hence depending on various investigations the final diagnosis of the Jaffe-Lichtenstein syndrome (JLS) was made and patient was referred to craniofacial unit for the further management.

\section{Conclusion}

The fibrous dysplasia affecting the craniofacial region is a distinctive entity that can, in most cases, be treated by conservative re contouring. The procedure is preferably indicated after the active growth phase has ceased. A longterm follow up of these patients is mandatory considering the probable flare up of continuous growth of the lesion.

\section{Conflict of Interest: None.}

\section{References}

1. Lichtenstein L. Polyostotic fi brous dysplasia. Arch Surg. 1938:36:874-898

2. Neville BW, Damm DD, Allan CM, Bouquot JE: Bone Pathology. Oral and Maxillofacial Pathology, 2nd edition. WB Saunders Company. 2002;635-640.

3. Ricalde P, Horswell BB. Craniofacial fi brous dysplasia of the fronto-orbital region: A case series and literature review. $J$ Oral Maxillofac Surg. 2001;59:157-167. 
4. Abdel-Wanis ME, Tsuchiya H: Melatonin deficiency and fibrous dysplasia: might a relation exist? Med Hypotheses. 2002;59:552-554.

5. Faves MJ, Vokes TJ: Paget disease and other dysplasias of bone.InHarrison's principles of Internal Medicine 16th edition. Edited by: Kasper DL, Braunwald E, Fauci AS, Hauser SL, Longo DL, Jameson JL. New York, McGraw-Hill. 2005;22792286.

6. Alam Wg, Cdr A, Chander GP, Capt BN: Craniofacial fibrous dysplasia presenting with visual impairment. MJAFI. 2003;59:342-343.

7. Yetiser S, Gonul E, Tosun F, Tasar M, Hidir Y: Monostotic craniofacial fibrous dysplasia: The Turkish experience. $J$ Craniofac. 2006;171:62-67.

8. Riminucci M, Saggio I, Robey PG, Bianco P: Fibrous dysplasia as a stem cell disease. $J$ Bone and Mineral Res. 2006;21:P125-P131.
9. Ogunsalu CO, Lewis A, Doonquah L: Benign fibro-osseous lesions of the jaw bones in Jamaica: analysis of 32 cases. Oral Dis. 2001;7:155-162.

10. Mohan RP, Verma S, Gupta N, Ghanta S, Agarwal N, Gupta S. The radiological versatility of fibrous dysplasia: An 8-year retrospective radiographic analysis in a north Indian population. Indian J Dent. 2014;5(3):139-145.

How to cite this article: Hameed S.K, Chatra L, Shenoy P, Mathew A K. Jaffe-Lichtenstein syndrome - Report of a rare case. J Oral Med, Oral Surg, Oral Pathol, Oral Radiol. 2018;4(4):194-197. 